\title{
Judicialização da saúde: gastos Federais para o Sistema Único de Saúde (SUS) entre 2011-2014
}

Lawsuits to receive free medication: Federal expenditures for the Brazilian Public Health System (SUS) between 2011-2014

Judicialización de la salud: gastos Federales para el Sistema Público de Salud entre 20112014

Karina Pires Nogueira ${ }^{1}$

Erika Barbosa Camargo ${ }^{2}$

RESUMO: Objetivo: analisar os gastos Federais para o sistema público de saúde Brasileiro com medicamentos obtidos por meio de ações judiciais entre os anos de 20112014. Metodologia: estudo transversal, com características descritivas e analíticas. Dados levantados na plataforma DW/COMPRASNET. Resultados: De 12.578 processos de judicialização na esfera federal foram extraídos 15 medicamentos com maior valor de aquisição, destes, 7 medicamentos corresponderam a $\mathrm{R} \$ 1,45$ bilhão do orçamento federal o que significou $87 \%$ do gasto total das ações estudadas. Dos $15,14,28 \%(n=4)$ tinham registro na Anvisa, estavam incorporados pela Comissão Nacional de Incorporação de Tecnologias no SUS (CONITEC) e eram integrantes da Relação de Medicamentos Essenciais (RENAME); 46,42\% $(n=13)$ possuíam registro na Anvisa, porém não incorporados pela CONITEC e não integrantes da RENAME; 3,57\% $(n=1)$ com registro na Anvisa, incorporados pela CONITEC e não integrantes da RENAME e 35,71\% ( $n=10)$ sem registro na Anvisa, não incorporados pela CONITEC e não integrantes da RENAME. Conclusão: Com a judicialização da saúde as aquisições são realizadas sem planejamento ou estabelecimento de critérios mínimos o que pode comprometer a sustentabilidade do SUS.

Palavras-chave: Judicialização. Decisões judiciais. Direito à saúde

ABSTRACT: Objective To analyze Federal expenditures for the Brazilian public health system with drugs obtained through lawsuits between the years 2011-2014. Methods: cross-sectional study, with descriptive and analytical characteristics. Data collected from the DW / COMPRASNET platform. Results: In total 12,578 lawsuits were identified at the federal level and 15 drugs with the highest acquisition value were extracted. Of these, seven drugs corresponded to US\$ 452.644.065.68 million dollars of the federal budget, which represented $87 \%$ of the total expenditure of the actions studied. Of the 15 drugs / year studied, $14.28 \%(n=4)$ were registered at the National Brazilian Surveillance Agency (ANVISA), were incorporated by the National Commission for the Incorporation of Technologies in SUS (CONITEC) and were part of the List of essential drugs (RENAME);

\footnotetext{
1 Secretaria de Ciência Tecnologia e Insumos Estratégicos/Departamento do Complexo Industrial e Inovação em Saúde, Ministério da Saúde. Email: karina.nogueira@saude.gov.br

2 Faculdade de Ciências da Saúde/Departamento de Nutrição, Universidade de Brasilia. Email: erika.barbosacamargo@gmail.com
} 
46.42\% ( $n=13$ ) were registered with ANVISA, but not incorporated by CONITEC and not members of RENAME; $3.57 \%(n=1)$ registered in ANVISA, incorporated by CONITEC and non-RENAME members and $35.71 \%(n=10)$ without ANVISA registration, not incorporated by CONITEC and not RENAME members. Conclusion: With the Lawsuits to receive free medication, the acquisitions are carried out without planning or establishing minimum criteria, may compromise SUS sustainability.

Key words: Judicialization. Judge Decisions. Right to Health

RESUMEN: Objetivo: analizar los gastos federales para el sistema público de salud brasileño con medicamentos obtenidos por medio de acciones judiciales entre los años 2011-2014. Metodología: estudio transversal, con características descriptivas y analíticas. Datos levantados en la plataforma DW / COMPRASNET. Resultados de 12.578 procesos de judicialización en la esfera federal fueron extraídos 15 medicamentos con mayor valor de adquisición, de éstos, 7 medicamentos correspondieron a $R$ \$1,45 mil millones del presupuesto federal lo que significó el $87 \%$ del gasto total de las acciones estudiadas. De los $15,14,28 \%(n=4)$ tenían registro en la Anvisa, estaban incorporados por la Comisión Nacional de Incorporación de Tecnologías en el SUS (CONITEC) y eran integrantes de la Relación de Medicamentos Esenciales (RENAME); 46,42\% ( $n=13$ ) tenían registro en Anvisa, pero no incorporados por la CONITEC y no integrantes de RENAME; EI 3,57\% $(n=$ 1) con registro en Anvisa, incorporados por la CONITEC y no integrantes de RENAME y el $35,71 \%(n=10)$ sin registro en la Anvisa, no incorporados por la CONITEC y no integrantes de RENAME. Conclusión: Con la judicialización de la salud las adquisiciones se realizan sin planificación o establecimiento de criterios mínimos lo que puede comprometer la sustentabilidad del SUS.

Palabras Ilave: Judicialización. Decisiones judiciales. Derecho a la salud

\section{Introdução}

A Judicialização da saúde vem sendo enfrentada em diversos países do mundo de forma distinta do Brasil. Em determinados países da Europa, nos quais os direitos coletivos se sobrepõem aos individuais, os produtos que não integram os protocolos clínicos não são cobertos pelos sistemas de saúde ou seguros de saúde. Diante desta condição, ações impetradas por advogados de indivíduos ou empresas na Europa não tem arcabouço para impor ao Estado o custeio de determinadas coberturas que não possuem eficácia e segurança comprovadas. Nos Estados Unidos, por força de sua estrutura de saúde estar concentrada na assistência por meio dos planos de saúde a compreensão é de que o estabelecido em seus contratos individuais deve prevalecer. Isto ocorre devido ao impacto considerável que as ações judiciais podem significar nos gastos setoriais (1).

No Brasil uma tendência de aumento de ações judiciais impetradas na esfera federal e também os Estados da federação vem sendo observada. Em âmbito federal, o aumento 
vertiginoso destas ações, desde 2006, alcançaram a marca de 1 bilhão de reais em 2015. Nos Estados, tomando alguns exemplos de demandas judiciais, principalmente destinadas ao tratamento de doenças oncológicas, foram identificados casos como da Bahia - que entre 2006 e 2007 o número de ações cresceu de 34 para 112 - e Santa Catarina - cujas ações cresceram de 24 para 2.511 entre 2002 e 2007 (2). Ainda, no estado de Pernambuco, o número de demandas praticamente dobrou entre 2009 e 2010, onde foram impetradas 655 ações para o período, sendo que 40,8\% destas eram destinadas ao tratamento de neoplasias (tumores) $(3,4)$.

Quando se emprega o termo judicialização da saúde, corriqueiramente utilizado por pessoas que recorrem ao Poder Judiciário para ter acesso a medicamentos, deve-se considerar que essa expressão está relacionada a problemas de acesso a bens, serviços, medicamentos, dentre outros (5). Considerado por alguns estudiosos como um "fenômeno", a judicialização persiste na reclamação de bens e serviços de saúde por qualquer cidadão brasileiro. E os objetos que mais compõe essas demandas judiciais são os medicamentos.

Legitimando a solidez e o fortalecimento do sistema judiciário brasileiro não se pode argumentar seu papel diante de casos de omissão do Governo ou não cumprimento ao estabelecido nas políticas de assistência à saúde. Diante deste fato, as ações judiciais contribuem na viabilidade do acesso imediato aos medicamentos que, conforme argumento jurídico, compõem direitos garantidos e que a sua indisponibilidade está associada a uma implementação ineficiente de assistência farmacêutica definida pelo Estado (6).

Entretanto, apesar da legitimidade da tutela judicial, não se pode deixar de destacar os impactos destas ações que são realizadas de forma deliberada e em confronto com a responsabilidade do Estado em prover os cidadãos de medicamentos seguros, eficazes e de qualidade. É imprescindível que o Estado responda ao proposto em suas atribuições em relação às necessidades de saúde (2).

Ainda, baseando-se nas lacunas apresentadas de descumprimento do proposto no direito constitucional, observa-se a importância de se avaliar a evolução do processo de judicialização no Brasil em nível Federal. Diante deste cenário, faz-se o questionamento: à judicialização da saúde pode inviabilizar a sustentabilidade do SUS? 
Em função da crescente demanda por judicialização da saúde no Brasil estima-se que o aumento dos custos pode inviabilizar a sustentabilidade do SUS. Pacientes sentem que suas necessidades por saúde não estão cobertas pelo SUS e cada vez mais recorrem ao poder judiciário para obter acesso a algum tipo de tratamento, mesmo não havendo evidências científicas sobre a segurança e eficácia dos medicamentos. Este contexto despertou o interesse em avaliar os medicamentos judicializados no Brasil (esfera federal) devido aos diferentes interesses dos atores envolvidos.

Visando subsidiar esta reflexão, objetivou-se avaliar os gastos com medicamentos oriundos de ações judiciais entre os anos de 2011 e 2014 impetradas contra o Governo Federal.

\section{Metodologia}

Foi realizado um estudo transversal, com características descritivas e analíticas, utilizando-se das informações identificadas nos levantamentos dos gastos com medicamentos oriundos de ações judiciais entre os anos de 2011-2014. Foram extraídos da plataforma DW/COMPRASNET dados dos perfis das aquisições realizadas como: produto, quantidade adquirida, valores unitários e totais, produtos mais demandados e de maior valor.

Foram analisadas num total 12.578 ações judiciais entre os anos de 2011-2014. Nos anos de 2011, 2012, 2013 e 2014 foram avaliadas respectivamente 2.891, 3.201, 3.032 e 3.454 ações judiciais. Durante a análise foram excluídos da amostra ações judiciais de equipamentos e materiais de uso em saúde, ficando o foco da análise somente para medicamentos. Para o cálculo amostral foi considerado um erro amostral de $10 \%$, nível de confiança de $90 \%$ totalizando uma análise de cerca de 15 ações de medicamentos (excluindo-se os medicamentos repetidos em diferentes ações). Deste escopo, deverão, ainda, ser avaliados os seguintes pontos:

1. Maior volume de recursos gastos por medicamento em cada ano;

2. Quantidade de medicamentos judicializados com maior frequência por ano;

3. Medicamentos judicializados que possuem registro na Anvisa;

4. Medicamentos judicializados que já estão incorporados, pela CONITE, ao SUS; 
5. Medicamentos judicializados que já estão incluídos na incluídos na RENAME.

\section{Resultados}

Foram elencados 12.578 processos de judicialização na esfera federal (entre 20112014). Deste total, foram extraídos os 15 medicamentos/ano, com maior impacto financeiro no volume total de recursos destinados às demandas judiciais. Em cada ano, dos 15 medicamentos 7 deles se repetiam 4 anos consecutivos, os outros 8 medicamentos ou eram inéditos ou não se repetiram 4 anos consecutivos. Ao consolidar os quatro anos, obteve-se um total de 28 medicamentos.

Foram levantados os gastos totais com judicialização, entre 2011 e 2014, contemplando todos os produtos adquiridos mediante ações judiciais impetradas contra a União (Figura I).

Figura I. Gastos totais da União com ações judiciais (2011-2014).

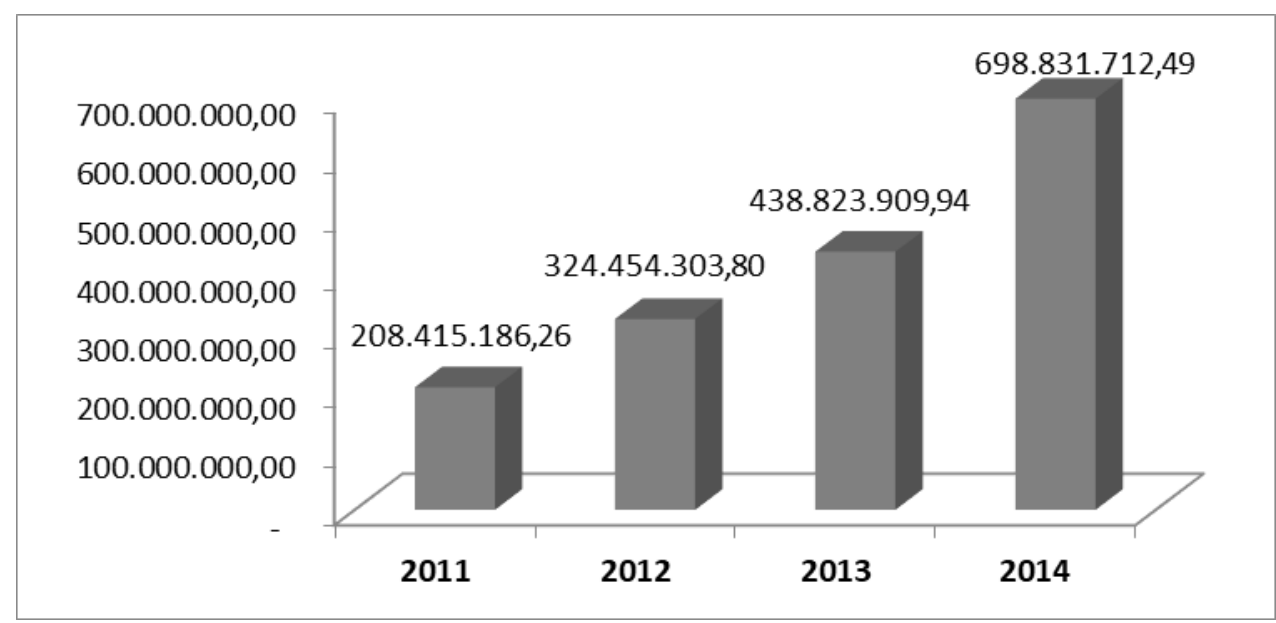

Fonte: DCJUR/DLOG/SE/MS

Concomitante ao aumento no volume de recursos destinados a estas ações está o número de processos ocorridos no período estudado. Os números totais, em cada ano mantém um volume aproximado, mas em escala crescente.

No período analisado, com extratos de 15 medicamentos/ano, encontrou-se o grande volume de destinação financeira em produtos com considerável valor de aquisição individual (acima de 350 mil reais/ano). Ao concentrar todos os medicamentos, incluindo os que se repetiram no período analisado em um total de 28 produtos, observou-se que a 
maioria destes medicamentos é destinada ao tratamento oncológico e de doenças raras (Tabela I).

Tabela I. Medicamentos e indicação com maior volume de gastos por meio de judicialização - Consolidado dos 15 medicamentos/ano (2011-2014).

\begin{tabular}{|c|c|}
\hline MEDICAMENTOS & INDICAÇÃO \\
\hline Abiraterona acetato, $250 \mathrm{mg}$ & Câncer de próstata \\
\hline $\begin{array}{l}\text { Alfa-1 antitripsina (AAT), } 20 \mathrm{mg} / \mathrm{ml} \text {, solução } \\
\mathrm{p} \text { / infusão injetável }\end{array}$ & Deficiência de Alfa-1 \\
\hline Alfagalsidase, $1 \mathrm{mg} / \mathrm{ml}$, solução $\mathrm{p} /$ infusão & Doença de Fabry \\
\hline Alfalglicosidase, 50 mg, pó liófilo p/ injetável & Doença de Pompe \\
\hline Betagalsidase, 35 mg, pó liófilo p/ injetável & Doença de Fabry \\
\hline Bosentana, $125 \mathrm{mg}$ & PCDT da Hipertensão Arterial Pulmonar \\
\hline $\begin{array}{l}\text { Brentuximabe Vedotina, } 50 \text { mg, pó liófilo p/ } \\
\text { injetável }\end{array}$ & Linforma de Hodgkin e LGCs \\
\hline Cetuximabe, $5 \mathrm{mg} / \mathrm{ml}$, solução injetável & Câncer colorretal \\
\hline Eculizumabe, $10 \mathrm{mg} / \mathrm{ml}$, solução injetável & Hemoglobinúria parxística noturna \\
\hline Elosulfase alfa, $1 \mathrm{mg} / \mathrm{ml}$, solução injetável & Mucopolissacaridose tipo IVA \\
\hline $\begin{array}{l}\text { Galsulfase, } 1 \mathrm{mg} / \mathrm{ml} \text {, concentrado para } \\
\text { perfusão injetável }\end{array}$ & Mucopolissacaridose tipo VI \\
\hline $\begin{array}{l}\text { Hemina, } 25 \mathrm{mg} / \mathrm{ml} \text {, concentrado } \mathrm{p} / \text { solução } \\
\text { para perfusão }\end{array}$ & $\begin{array}{llc}\text { Porfiria Aguda } & \text { Intermitente/Porfiria } \\
\text { Variegata/Coproporfiria Hereditária }\end{array}$ \\
\hline Hemina, 313 mg, injetável & Porfiria. Deficiência de heme no fígado \\
\hline $\begin{array}{l}\text { Idursulfase, } 2 \mathrm{mg} / \mathrm{ml} \text {, solução } \mathrm{p} / \text { infusão } \\
\text { venosa }\end{array}$ & Síndrome de Hunter (Mucopolissicaridose II) \\
\hline $\begin{array}{l}\text { Inibidor de esterase, inibidor de esterase c1 } \\
\text { humana, } 500 \text { ui, pó liófilo para injetável }\end{array}$ & Angioma Hereditário \\
\hline Laronidase, $0,58 \mathrm{mg} / \mathrm{ml}$, solução injetável & Mucopolissacaridose tipo I \\
\hline Lomitapida, $10 \mathrm{mg}$ & Doenças cardiovasculares \\
\hline Lomitapida, $5 \mathrm{mg}$ & Doenças cardiovasculares \\
\hline Maraviroque, $150 \mathrm{mg}$ & Infecção pelo HIV \\
\hline Miglustate, $100 \mathrm{mg}$ & PCDT da Doença de Gaucher \\
\hline Rituximabe, $10 \mathrm{mg} / \mathrm{ml}$, solução injetável & $\begin{array}{l}\text { Linforma não Hodgkin/ Artrite Reumatóide/ } \\
\text { Leucemia Linfóide Crônica/ Granulomatose } \\
\text { de Wegener }\end{array}$ \\
\hline Sorafenibe tosilato, $200 \mathrm{mg}$ & Carcinoma \\
\hline Sunitinibe, Malato, $50 \mathrm{mg}$ & $\begin{array}{l}\text { Tumor Gastrointestinal/ Carcinoma Renal/ } \\
\text { Tumor Pancreático }\end{array}$ \\
\hline Tafamidis, $20 \mathrm{mg}$ & Polineuropatia Amiloidótica \\
\hline Tocilizumabe, 20 mg/ml, solução injetável & PCDT da Artrite Reumatóide \\
\hline Trastuzumabe, 440 mg, pó liofilo injetável & Câncer de mama mestastático \\
\hline
\end{tabular}


Brentuximabe Vedotina, $50 \mathrm{mg}$, pó liófilo p/ Linfoma de Hodgkin injetável

\begin{tabular}{l|l}
\hline Carfilzomib, 60 mg, pó liófilo p/ injetável & Mieloma Múltiplo \\
\hline
\end{tabular}

A participação de medicamentos oncológicos e para doenças raras nos volumes totais de recursos destinados às demandas jurídicas foi considerada relevante, permitindo sinalizar para a importância de se avaliar mais profundamente o uso desta modalidade no atendimento à população (Figura II).

Figura 2. Participação total dos 15 medicamentos/ano com maior volume de gastos por meio de judicialização (2011-2014).

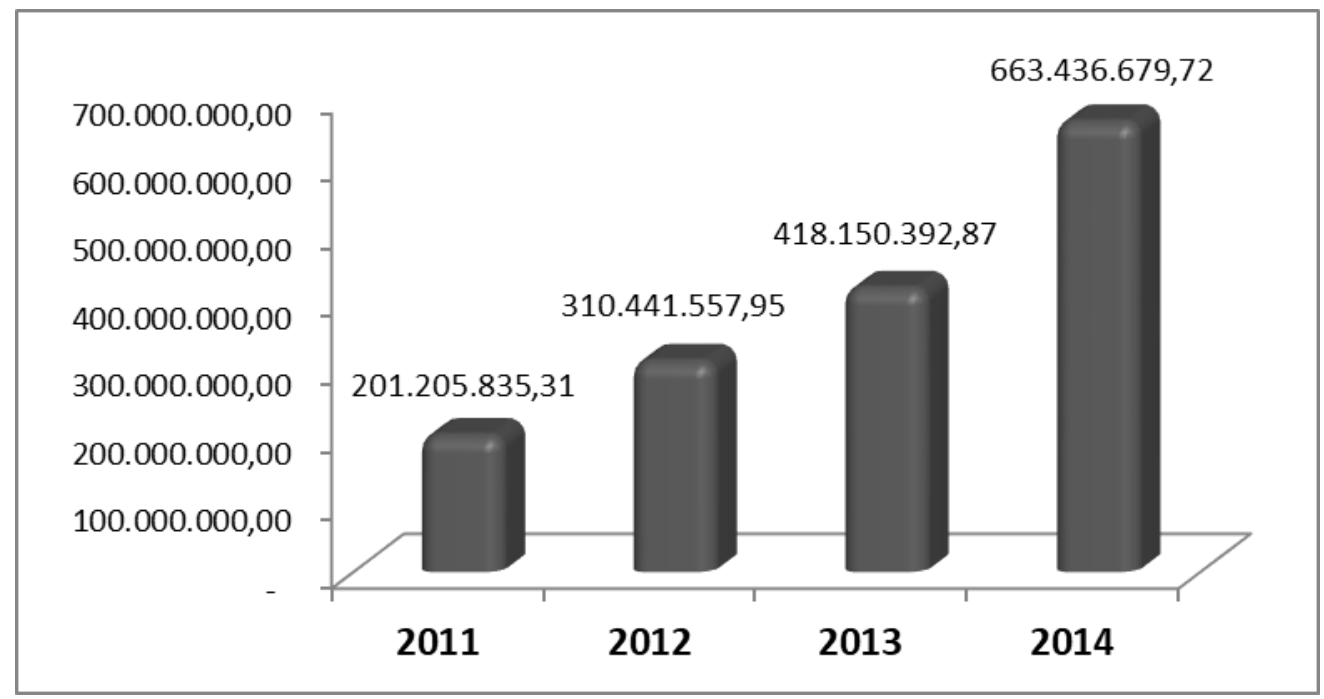

Fonte: DCJUR/DLOG/SE/MS

Ainda, dos de 15 medicamentos/ano foram identificados 7 medicamentos (Alfagalsidase, Alfaglicosidase, Betagalsidase, Eculizumabe, Galsulfase, Idursulfase e Laronidase) que foram objeto de ação judicial nos quatro anos analisados (2011-2014). Estes 7 medicamentos corresponderam a $R \$ 1,45$ bilhão do orçamento federal no período o que significou $87 \%$ do gasto total das ações estudadas.

É imperativo salientar que os medicamentos analisados permitiram identificar o perfil de cada um no que se refere a compor ao menos uma das três recomendações (registro na Anvisa, incorporação pela CONITEC e inclusão na RENAME), tornando a sentença 
judicial apropriada aos critérios do Ministério da Saúde. Nota-se um baixo percentual do que é classificada como exigência mínima para os padrões de segurança, eficácia, efetividade, eficiência e impactos econômicos (Tabela II).

Tabela 2. Perfil dos medicamentos (Consolidado dos 15 medicamentos/ano) quanto ao registro, incorporação e relação de medicamentos essenciais.

\begin{tabular}{|c|c|}
\hline $\begin{array}{l}\text { Produtos com registro na Anvisa, } \\
\text { incorporados pela CONITEC e integrantes } \\
\text { da RENAME. }\end{array}$ & $\begin{array}{c}14,28 \% \\
\text { (4 medicamentos) }\end{array}$ \\
\hline $\begin{array}{l}\text { Produtos com registro na Anvisa, não } \\
\text { incorporados pela CONITEC e não } \\
\text { integrantes da RENAME. }\end{array}$ & $\begin{array}{c}46,42 \% \\
\text { (13 medicamentos) }\end{array}$ \\
\hline $\begin{array}{l}\text { Produtos com registro na Anvisa, } \\
\text { incorporados pela CONITEC e não } \\
\text { integrantes da RENAME. }\end{array}$ & $\begin{array}{c}3,57 \% \\
\text { (1 medicamento) }\end{array}$ \\
\hline $\begin{array}{l}\text { Produtos sem registro na Anvisa, não } \\
\text { incorporados pela CONITEC e não } \\
\text { integrantes da RENAME. }\end{array}$ & $\begin{array}{c}35,71 \% \\
(10 \text { medicamentos })\end{array}$ \\
\hline
\end{tabular}

\section{Discussão}

Esta avaliação tem como ponto de partida o número excessivo de ações judiciais por ano, onde o Estado é parte condenada a cumprir as sentenças avaliadas pelo Poder Judiciário, não havendo, por muitas vezes, possibilidade de exercer o princípio da economicidade nas aquisições realizadas para atender em tempo hábil o escopo da ação. Para Sant'Ana (6) "a promoção do acesso a um bem ou serviço em saúde relaciona-se, dentre outros fatores, ao aporte de recursos financeiros alocados para esse fim".

Dos medicamentos analisados - extraídos de 12.578 processos no período de 2011 2014 - destacados neste estudo permitiu-se observar o alto volume de recursos destinados a estas ações, confrontando diretamente o princípio da economicidade. De um total de 28 produtos, alerta-se para o fato de que 7 destes produtos, judicializados em todos os anos do período estudado, corresponderam a uma crescente participação no volume total do comprometimento orçamentário. Já neste extrato realizado é possível identificar claramente o impacto que estes medicamentos ocasionam e, em sua maioria, sem justificativas técnico-científicas para continuidade destas aquisições. As quantidades totais 
adquiridas, apesar de manterem uma aproximação entre os anos, também estão em uma escala crescente.

Quando se trata de destinação terapêutica, o trabalho apresenta um número considerável de produtos destinados ao tratamento de doenças oncológicas e doenças raras. Cabe destacar que, para a OMS, doenças raras são aquelas que afetam até 65 pessoas a cada 100 mil indivíduos. No extrato de análise de 28 produtos, foram identificados 12 medicamentos para tratamento de doenças raras e 9 destinados ao tratamento oncológico.

Em complemento, destaca-se a elevada participação dos 7 medicamentos mencionados, correspondendo a $87 \%$ do orçamento total destinado às ações judiciais realizadas em âmbito federal no período de 2011 a 2014.

Ante ao exposto, cabe reiterar que o processo de análise pelas esferas jurídicas de cumprimento do direito exercido pelo indivíduo é conflitante com o previsto nas ações orçamentárias programadas anualmente para pleno atendimento igualitário nas políticas de saúde. Esta condição gera um dissenso entre o que é priorizado pelos órgãos responsáveis pela execução das políticas de saúde como um direito coletivo com ações jurídicas individuais. Para Mazza (7), o Poder Judiciário não pode deixar de tutelar os direitos fundamentais, entretanto não podem causar danos aos direitos coletivos.

Consoante a esta condição, em uma análise realizada pela Advocacia Geral da União (AGU), em conjunto com a Consultoria Jurídica do Ministério da Saúde (CONJUR/MS), há claramente o destaque do alto custo "muito mais significativo e imensurável do ponto de vista econômico" quanto à concorrência das compras realizadas pelo Governo Federal para abastecimento do SUS e o cumprimento de sentenças judiciais.

Segundo D’Espíndula (5), por muitas vezes são produtos sem registro que receberam pareceres desfavoráveis da CONITEC. Esta modalidade da forma como se apresenta descarta critérios de segurança, eficiência e eficácia dos produtos que, obrigatoriamente, o ente federado tem que disponibilizar ao demandante. Além de submeter ao tratamento produtos sem uma avaliação específica e determinada em legislações internacionais, esse tipo de compra impacta diretamente no orçamento público.

Diante do avanço destas ações e de uma possível superficialidade nas análises, em 2010 o Conselho Nacional de Justiça (CNJ) apresentou a Recomendação 31/2010 (8), direcionada aos Tribunais de Justiça dos Estados e aos Tribunais Regionais Federais com 
objetivo de auxiliar os operadores do direito na construção das sentenças assegurando maior eficiência nas questões de saúde.

Nota-se, desta forma, que o Governo Federal tem mantido a judicialização da saúde como pauta permanente, visando um encaminhamento que possibilite a sua redução diante dos já tão divulgados impactos em seu orçamento. No Relatório de Levantamento FiscSaúde (9), apresentado pelo Tribunal de Contas da União (TCU), aqueles apontamentos são apresentados com rigor na análise da execução orçamentária do Ministério da Saúde.

A importância no cumprimento dos fundamentos básicos para a prescrição de um medicamento como registro na Anvisa, disponibilidade no SUS, alternativa terapêutica, estudo da medicação junto à CONITEC, informações se o paciente é usuário de plano privado de saúde, bem como dados sobre possíveis conflitos de interesse do profissional que prescreveu a receita, foi registrado no Parecer no 810/2012 AGU/CONJUR-MS (10) e referenciado pelo TCU em seu Relatório.

Apesar desta instrução de 2012, estas situações irregulares foram apresentadas novamente em 2016 no referido documento daquele tribunal de contas. E novamente foi destacado que as ações judiciais sobrecarregam o orçamento público ao tempo em que desconsideram o sistema de saúde existente.

Contudo, por força de determinação legal, as aquisições são realizadas de forma individualizada e com prazo exíguo para cumprimento dos princípios da economicidade, sem planejamento ou estabelecimento de maiores critérios. Reforça-se aqui o desvio da destinação dos recursos a políticas estratégicas de saúde para estas ações, causando além de uma distorção nos serviços do SUS com uma redução no poder de compra do estado.

Observa-se que diversos fatores permeiam a questão dos gastos com judicialização em saúde para o SUS. A interferência das determinações judiciais no orçamento do Estado além de distanciar o papel fundamental de atendimento a um direito coletivo, ainda impõe um gasto sem qualquer planejamento ou análise financeira pelo órgão judicializado, impedindo a otimização de seus recursos e inviabilizando a sua melhor aplicação em produtos e serviços de saúde. 


\section{Conclusão}

Diante do que se observa em diversos países do mundo, onde podem ser identificados possíveis casos de judicialização em saúde, percebe-se claramente que a abordagem realizada difere do que tem ocorrido no Brasil em uma progressão considerável. Em países onde o sistema de saúde se aproxima do brasileiro, é possível observar que a priorização do acesso aos produtos e serviços disponíveis é focada no coletivo e não pontuda em atendimento individualizado, fato este que compromete quaisquer modalidades judiciais que possam imprimir outra destinação de recursos.

Objetiva-se não comprometer orçamentos previamente planejados com ações judiciais pontuais que contemplem um indivíduo ou grupo específico. Esta condição é verificada até em países onde o sistema de saúde não é gratuito, fomentado pelo Governo. No caso americano a assistência é claramente definida em seus contratos de planos de saúde, minimizando possíveis gastos em questões judiciais.

No Brasil, apesar da legitimidade de um Sistema Único de Saúde que tem como diretrizes básicas a equidade, universalidade e integralidade no acesso a seus produtos e serviços, o crescimento de ações judiciais, principalmente tendo medicamentos como objetivos, vem comprometendo o Governo Federal, Estados da Federação e Municípios. Percebe-se uma crescente orientação para a judicialização da saúde, visando acelerar uma assistência de responsabilidade do Estado de forma individualizada, sem considerar a eficiência terapêutica, os custos para o sistema e impactos sociais. Nos anos analisados neste trabalho, este crescimento minimamente dobrou em número de processos que, por conseguinte, implicou diretamente em seus orçamentos.

Além desta preocupação com o impacto financeiro no orçamento público e na ocorrência da iniquidade no acesso aos medicamentos outras questões devem ser tratadas com a importância que recai sobre elas. Muitos medicamentos judicializados não possuem registro junto à Anvisa, não são incorporados pela CONITEC e, por fim, não compõem a lista da RENAME.

Diversos órgãos públicos que tem como responsabilidade institucional o controle, a análise jurídica e o funcionamento do Estado e de suas políticas públicas se debruçaram sobre tema da judicialização da saúde. Além de alertar para o crescimento destas ações e seus impactos financeiros também avaliaram os papeis de cada ente envolvido. 
A manutenção do estado e de suas políticas e programas voltados para o atendimento da população dependem de recursos financeiros, mas também de estratégias de atuação e ambiente propício para implementar o que se propõe. Atualmente, o sistema de saúde brasileiro vem sofrendo continuamente estas interferências das demandas judiciais. Contudo, o que mais exige uma reflexão é o fato de que mesmo com um arcabouço legal, orientado pela Organização Mundial de Saúde, bem como um portfólio de padrões técnico-científicos vigentes estas condições não impedem a reincidência de demandas judiciais inapropriadas que distorcem o objetivo principal do pleno acesso aos produtos e serviços do SUS.

\section{Referências}

1. Medici AC. Judicialização , integralidade e financiamento da saúde. Med Baseada em Evidências. 2010;15(2):81-7.

2. Soares JCR de S, Deprá AS. Ligações perigosas: Indústria farmacêutica, associações de pacientes e as batalhas judiciais por acesso a medicamentos. Physis. 2012;22(1):311-29.

3. Campello, Bernadete Santos, Beatriz Valadares Cendón and JMK. Fontes de informação para pesquisadores e profissionais. Belo Horizonte: Editora UFMG; 2007.

4. Marçal K. A Judicialização da Assistência Farmacêutica: o caso Pernambuco em 2009 e 2010. 2012; Disponível em http://www.cpqam.fiocruz.br/bibpdf/2012marcal-kks.pdf [ Acesso em 30 maio 2017 ]

5. D’Espíndula T. Judicialização da medicina no acesso a medicamentos: reflexões bioéticas. Rev bioét(Impr) . 2013 Disponível em:

http://pesquisa.bvsalud.org/oncologiauy/resource/en/lil-704222 [Acesso em 30 maio 2017] ;

6. Sant'Ana J, Pepe V, Osorio-de-Castro C. Essencialidade e assistência farmacêutica: considerações sobre o acesso a medicamentos mediante ações judiciais no Brasil. 2011; Disponível em: http://iris.paho.org/xmlui/handle/123456789/9561 [Acesso em 30 maio 2017]]

7. Mazza F. Os impasses entre a judicialização da saúde e o processo orçamentário sob a responsabilidade fiscal: uma análise dos fundamentos decisórios do Supremo Tribunal. 2013; Disponível em http://pesquisa.bvsalud.org/enfermeria/resource/es/lil713153 [ Acesso em 30 maio de 2017]

8. Conselho Nacional de Justiça. Atos Normativos. Recomendacao n 31. 2010. p. 4. 
Cuadernos Iberomericanos

de Derecho Sanitario

9. Palmeira G. Tribunal de contas da união. Disponível em: file://C:/Users/User/Documents/Artigo Karina/002.142.2015-5_FiscSaude_.pdf [ Acesso em 30 maio 2017]

10. União ADA, Jurídica C, Ao J, Da M. Advocacia-Geral da União. 2012;

Recebido em: 16.6.2017 Aprovado em: 29.6.2017 